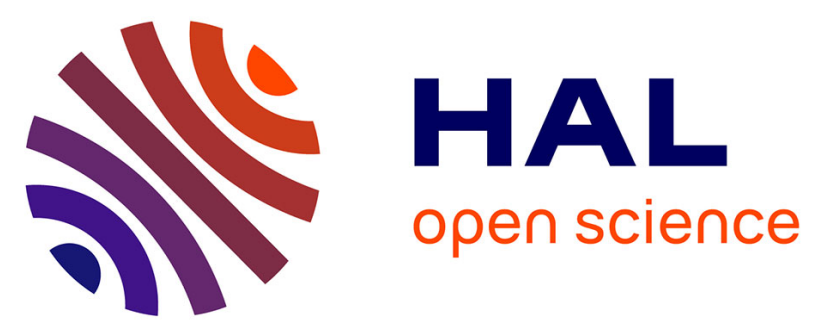

\title{
Eschyle et Euripide entre tragédie et comédie: polyphonie et interprétation dans quelques traductions récentes des Grenouilles d'Aristophane
}

Ariane Eissen, Myrto Gondicas

\section{To cite this version:}

Ariane Eissen, Myrto Gondicas. Eschyle et Euripide entre tragédie et comédie: polyphonie et interprétation dans quelques traductions récentes des Grenouilles d'Aristophane. Olson, S. Douglas. Ancient Comedy and Reception. Essays in Honor of Jeffrey Henderson, De Gruyter, pp.1022-1039, 2013, 9781614511250. 10.1515/9781614511250.1022 . hal-01648957

\section{HAL Id: hal-01648957 \\ https://hal.science/hal-01648957}

Submitted on 12 Dec 2017

HAL is a multi-disciplinary open access archive for the deposit and dissemination of scientific research documents, whether they are published or not. The documents may come from teaching and research institutions in France or abroad, or from public or private research centers.
L'archive ouverte pluridisciplinaire HAL, est destinée au dépôt et à la diffusion de documents scientifiques de niveau recherche, publiés ou non, émanant des établissements d'enseignement et de recherche français ou étrangers, des laboratoires publics ou privés. 
Ariane Eissen and Myrto Gondicas

\title{
Eschyle et Euripide entre tragédie et comédie: polyphonie et interprétation dans quelques traductions récentes des Grenouilles d'Aristophane
}

\begin{abstract}
The confrontation between Aeschylus and Euripides in Aristophanes' Frogs presents the translator with several challenges. Can he/she make clear the intrusion of the language of tragedy into that of comedy and convey the stylistic differences between the two dramatists? How should he/she interpret the pastiches at a second level of enunciation: is Aristophanes imitating in order to mock or to pay homage? To what extent should the translator point out the seriousness of the criticism or the gratuitousness of the clownery? And at an initial level of enunciation, can we really decide whom the umpire of the poetic joust, Dionysus, favors? There are also difficulties inherent in any translation of a play, such as suggesting a mise en scène or spelling out cultural elements obscured by the passage of time. This comparative study of four recent translations of Frogs (into English, French and Italian) aims to show how a translation/interpretation must come to grips with two languages, that of the translator and the Ancient Greek Aristophanes re-invents with consummate artistry.
\end{abstract}

La question de la paratragédie ${ }^{1}$ se trouve, par définition, à l'intersection de deux genres, dont elle permet d'explorer les rapports: rivalité, jeu, imitation ... Dans le cas des Grenouilles, le phénomène est mis au centre de la pièce puisque l'auteur comique fait s'affronter, avec comme arbitre le dieu du théâtre, deux poètes tragiques. On voit d'emblée la complexité d'un dispositif où la parole tragique (ou plutôt, deux types différents de parole tragique) sont manipulés de façon à « faire comédie ». Constamment, le texte comique laisse apparaître son double, l'univers tragique, dans un écart qui donne à rire; inversement, la tragédie est comicisée, amenée sur le terrain de la comédie, les deux genres jouant en alternance de leur caractère irréductible ou de la

Les questions soulevées dans ce texte ont fait l'objet d'une présentation orale au séminaire de Monique Trédé (Ecole Normale Supérieure, Paris) sur la comédie ancienne; le débat qui a suivi nous a aidées à préciser plusieurs points. Nous remercions chaleureusement Rossella Saetta Cottone de sa lecture attentive et de ses suggestions. Par ailleurs, la date à laquelle a été rédigée cette étude ne nous a pas permis de tenir compte du récent article de James Robson, « Transposing Aristophanes: The Theory and Practice of Translating Aristophanic Lyric », Greece and Rome 59 (2012), pp. 214-44. Mais nous tenions à en rappeler l'existence.

1 Sur laquelle on renverra toujours en premier lieu à l'ouvrage fondamental de Peter Rau, Paratragodia. Untersuchung einer komischen Form des Aristophanes, « Zetemata » 45 (Munich: Beck, 1967). 
possibilité d'une rencontre. La conséquence est bien évidemment une polyphonie accrue, qui met doublement au défi le traducteur. Défi stylistique: il faut rendre l'hétérogénéité des langages et des références. Défi interprétatif: il n'est pas toujours aisé de déterminer le statut (ironique ou non) d'un énoncé, voire de repérer le sens d'un mot (paratragique, purement comique?).

Nous nous sommes donc demandé comment un petit nombre de traducteurs récents s'étaient affrontés à ces difficultés; voici le corpus retenu pour cette enquête:

- Dario Del Corno, Aristofane. Le Rane : édition, texte grec, traduction, commentaire) (Milan: Fondazione Lorenzo Valla, Mondadori, 1985)

- Pascal Thiercy, Aristophane. Théâtre complet : édition, présentation, notes (Paris: Gallimard, « Bibliothèque de la Pléiade », 1997)

- Jeffrey Henderson (ed. et trans.), Aristophanes. Frogs [...] (Cambridge, Mass. / Londres: Harvard, collection « Loeb », 2002)

- Pierre Judet de La Combe, Aristophane. Les Grenouilles : édition, présentation, commentaire (Paris: Les Belles Lettres, collection « Budé », 2012). ${ }^{2}$

Nous examinerons divers aspects de la polyphonie dans les Grenouilles: le heurt et le mélange des tons; l'irruption du langage comme moyen et surtout comme enjeu du conflit entre les poètes; les problèmes herméneutiques pour déterminer la position d'Aristophane derrière la multiplicité des voix et des discours.

\section{De la réalité à la fiction: le plaisir de la reconnaissance}

Pour le lecteur d'aujourd'hui, pour l'auditeur du temps d'Aristophane, certains passages, dans ses comédies, se détachent nettement par un ensemble de traits déviants: vocabulaire (mots élevés, noms de héros, thématique du destin, de la perte et de la destruction...), syntaxe (complexité plus grande, usage de certaines formes d'adresse rituelles...), mètre (nombre moins élevé de résolutions dans le dialogue). D’ailleurs, sur la scène attique, le jeu théâtral devait souligner cet effet de "décrochage » et susciter le rire chez les spectateurs moins avertis, comme le suppose à juste titre K. J. Dover. ${ }^{3}$ Ces passages truffent la comédie d'une langue "paratragique " sans toujours se référer à une œuvre précise ni à un poète particulier. ${ }^{4}$ Quelle langue, dans

2 Nous désignerons désormais chaque auteur par ses initiales: DC, PT, JH, PJ. La numérotation des vers renvoie à l'édition de K.J. Dover citée infra, n. 3.

3 K.J. Dover (ed.), Aristophanes. Frogs (Oxford: Clarendon Press, 1993), p. 34.

4 Cependant, comme le note Rau, Paratragodia (ci-dessus, note 1), pp. 122-3, le thème des Grenouilles fait qu'on y trouve essentiellement, en matière paratragique, des passages inspirés d'Eschyle ou d'Euripide, en dehors même des citations littérales dans la scène de la pesée. 
les traductions contemporaines prises ici en exemple, les auteurs élaborent-ils pour rendre compte de ce phénomène?

Le vocabulaire (niveau de langue différencié, créations verbales) est sans doute le moyen le plus communément utilisé pour signaler la parodie, car cela suppose une intervention minimale (un mot plutôt qu'un autre), même s'il y faut de l'invention. Le chœur, au v. 814, décrit ainsi Eschyle à l'aide d'un mot emprunté à l'épopée: eribremetas. JH rend sensible le décalage du niveau de langue avec « mighty thunderer »; DC crée un mot poétique, " altitonante », sur le modèle de « altisonante »; PT explique en note son néologisme « le Rugitonnant », tandis que « le grand fracassant » de PJ est une réussite phonique, mais sémantiquement un peu éloignée.

Des traits spécifiques de syntaxe sont, ou non, restitués dans les traductions. Prenons le v. 1171 (tiré des Choéphores), où l'invocation se construit directement avec un deux infinitifs de but (kèrussô... kluein, akousai): chez JH, il semble que le marquage syntaxique soit déplacé sur le plan lexical, par l'emploi d'un verbe archaïque (I invoke my father, to hearken and hear), alors que les trois autres traducteurs trouvent des tours qui évoquent la brièveté remarquable de la construction grecque. Ainsi DC (proclamo al padre / di sentirmi, di ascoltarmi); très proche de lui, PT (Je clame à mon père / de m'entendre, de m'écouter); PJ fait des deux verbes d'écoute des propositions indépendantes, ce qui introduit une vivacité bien venue (J'invoque mon père, / Qu'il écoute, qu'il entende!).

Le rythme plus resserré (à cause de la relative rareté des résolutions) du trimètre tragique semble présenter un défi dans des traductions en prose ou en vers non mesurés. Comment rendre cette légère variation? JH, assez souvent, adopte le moule du blank verse shakespearien, par exemple: v. 1182, 1217-18, 1382, 1383, 1425 (parodie d'Ion de Chios), 1471 (d'après Euripide), 1475 (id.). ${ }^{5}$ Cependant, ce choix est très loin d'être systématique, si bien qu'on a un peu l'impression que le traducteur a accueilli d'heureuses trouvailles plutôt que délibérément visé un effet précis. DC ne semble pas rechercher de régularité particulière, il en est de même de PJ. On trouve chez PT un petit nombre d'alexandrins blancs, comme aux v. 840 (à condition de ne pas compter l'e muet) ou 1128; mais pas une seule fois dans la scène de la pesée, qui s'y prêterait le mieux puisqu'elle offre par principe des vers entiers et isolés de leur contexte. Là encore, on s'interroge sur la part respective du hasard et du choix.

Il arrive parfois que des vers paratragiques (reconnus comme citations grâce aux scholies ou parce que nous les avons par ailleurs) soient stylistiquement peu marqués; c'est surtout le cas avec ceux d'Euripide. Ainsi " coup de dés » d'Achille, au v. 1400, qui offre un vocabulaire quotidien (même s'il s'agit dans l'original d'une métaphore), une syntaxe non marquée et une résolution métrique (anapeste au troisième pied). DC («Due e quattro ha tirato Achille ») inverse le verbe et le sujet, trait du style élevé; PJ (« Il jeta, Achille, ... ») reprend le pronom sujet par le nom

5 Les vers cités sont bien entendu choisis parmi ceux qui ne présentent pas ou très peu de résolutions. 
propre, ce qui donne un effet étrange: la cacophonie du hiatus est-elle délibérée? JH ne propose aucun marquage rythmique ou stylistique; PT non plus, sauf à entendre le décasyllabe blanc (avec coupe irrégulière, au cinquième pied): « Achille a jeté deux as et un quatre. " Ici on a l'impression que les deux premiers ont voulu jouer du contraste entre un " moule " paratragique perceptible et la trivialité de l'image, tandis que les deux autres étendaient la banalité à tous les niveaux de la langue.

Quoi qu'il en soit, ces vers ou passages sont d'autant mieux sentis comme spécifiques qu'ils s'opposent stylistiquement au contexte où ils apparaissent. Un des traits du langage comique est la vivacité familière. Les stratégies des traducteurs, à cet égard, sont bien distinctes. DC est très souvent vif, tout en recherchant une certaine simplicité fluide: dans cet esprit, il n'hésite pas à introduire des variations syntaxiques, à couper les phrases de l'original (ainsi aux v. 757-8, les kai sont rendus par une construction différenciée; même chose aux v. 785-6); ailleurs, la divinité euripidéenne, le « gond » (strophinx) de la langue, au v. 892, devient, avec affaiblissement de l'image par changement syntaxique, « lingua mia roteante ». Est-ce aussi dans ce souci de simplicité qu'il recourt à des expressions lexicalisées, au lieu de chercher à coller aux images d'Aristophane? On citera les v. 1089-90, où la métaphore « sécher de rire " (aphauanthèn (...) gelôn) est quelque peu banalisée en " quasi scoppiavo dalle risa "; ou les " chansons de tireur de corde de puits » (himoniostrophou melè, v. 1297), rendues par «queste canzoni da facchino ». Autre trait simplificateur: la répétition d'un même terme ou d'une même tournure là où il y a variation en grec, ce qui donne un effet de martèlement ou d'écho assez efficace, mais au prix d'une déperdition des nuances ou des trouvailles verbales (« disgraziato » traduit tantôt $\hat{o}$ skhetlie andrôn au v. 1049, tantôt ô kakodaimon au v. 1058; "facitore di bravate a vanvera » glose le mot forgé kompophakellorrèmona, «che parlano a vanvera » en fait autant pour stômullomenôn au v. 1071). Côté rythme, il montre un grand souci de la mise en scène des dialogues, introduisant des mots à valeur logique et affective (anzi, allora, su, ma guarda, etc.) qui font bien sentir les positions respectives des personnages et les élans qui sous-tendent leurs prises de parole. Il surtraduit parfois: rhèma devient « parolone » (v. 854, 940), les deux fois à propos d'Eschyle, dont l'image de guerrier massif et bruyant est ainsi accentuée, voire extrapolée; dans la monodie parodique à la manière d'Euripide, au v. 1334, psukhèn apsukhon ekhonta devient « araldo dell'anima inanimata ».

Chez PT, le dialogue s'anime à l'aide de mots brefs qui donnent de l'élan (allons, penses-tu, dis donc, etc.) ou par d'autres traits du langage oral, comme l'usage d'un adjectif en fonction d'adverbe (v. 1221: « Cette burette va souffler dur »), ou l'effacement d'un verbe, sous-entendu dans une réponse (v. 1201: tu vas me les...?, où l'aposiopèse calque heureusement celle de l'original). Dans les vocatifs, souvent injurieux, il montre une tendance à l'argot, d'une sorte volontiers légèrement datée (v. 918, ho deina = "machin »; v. 921 ô panponéros = "l'archicanaille »; v. 933, ômathestat' = « bougre d'imbécile »), à moins qu'il n'opte pour un registre littéraire, là aussi un peu vieilli (au v. 1049, ô skhetli' andrôn donne " pitoyable individu », avec 
une nuance de distance ironique là où DC voit une injure violente: « disgraziato », de même que JH avec " you bastard ", alors que le " ô terreur » de PJ détone; dans le même style, on trouve " espèce de babillard », au v. 1160, pour ô katestôlmumene). Est-ce la crainte d'ennuyer par monotonie? PT oscille, pour la traduction des jurons, entre une adaptation parfois archaïsante (v. 795, "pardieu »; v. 931, 980, " pardieux »; v. 1045, " Grand dieu », où la minuscule initiale a la lourde charge de récuser toute connotation chrétienne) et des traductions littérales («par Apollon », au v. 952 ou « par Dèmèter », au v. 1067).

JH lance le dialogue grâce à de nombreux mots brefs ou tournures dynamiques (" so why...? ", « so what...? », " that's right », " not him! », " you see,... », " I'd guess that... »); il se sert généreusement d'expressions de la langue parlée: v. 765, manthanô = «I get the picture »; v. 795, Né Di' oligon husteron = « Yes, pretty soon »; v. 926, oimoi talas = "good grief! », etc. Ce goût pour l'oralité peut aller jusqu'à l'argot: v. 970, ou Khios = « not a bust after all », transposition du jeu de mots rendue possible par la note explicative jointe au passage; v. 1071, stômullomenôn = « as they gab away » v. 1197, lèreis = " hogwash. » Il y ajoute un procédé très anglo-saxon, et qui n'est pas exclusivement comique: l'allitération, où il se montre particulièrement inventif, comme aux v. 781 (ouranion g'hoson = to high heaven), 846 (ton khôlopoion = " this creator of cripples »), 922 (skordinai kai dusphoreis = fussing and fidgeting), 1160 (ô katestôlmumene = « you fool for folderol », avec un mot rare, archaïsant), etc.

On trouve chez PJ, dans le jeu des répliques, des tournures de la langue parlée contemporaine: " Ne dis pas ça! », « Comment ça? », " Mais si c'est ça »; des interrogations directes sans inversion: « Et c'est qui (...)? », « Ils vont faire des briques? »; pour les jurons, une vigoureuse adaptation (v. 749, « nom de Zeus! ») voisine avec une traduction littérale (v. 781, « par Zeus »). Assez souvent, il semble qu'une volonté de littéralité (née peut-être d'une lecture paratragique systématisée) aboutisse à des résultats bizarres, en particulier dans les vocatifs. Ainsi au v. 835, Dionysos appelle Eschyle ô daimoni' andrôn, en une tournure ironique, mais familière, qui rappelle les dialogues de Platon, ce qui donne "ô cher homme divin "; au v. 851, où le dieu l'appelle ô polutimèt' Aiskhule, on a " ô Eschyle mille fois adoré », introduisant une distance ironique un peu appuyée, là où DC et PT semblent comprendre l'expression au premier degré (« Eschilo veneratissimo », " Eschyle tant révéré »), tandis que JH est plus léger dans le paratragique shakespearien ( my exalted Aeschylus »). Cette tendance mène parfois à la surtraduction: v. 891, « tes petits dieux personnels », où " petits » est ajouté; v. 918, ho deina = « ce qui qu'il soit », tour bizarre qui rate le ton familier; v. 921, ô panponèros = « oh le totalement salaud », avec création syntaxique là où le grec a un mot relativement courant.

La langue de la comédie est émaillée de références à des réalités concrètes, datées. Qu'en font les traducteurs, entre littéralité et modernisation? Prenons l'exemple de la course à pied où les oliviers servent de bornes (v. 995): PJ colle à l'original («Que [...]/ l'ardeur [...]/ ne t'emporte pas loin des oliviers »); les autres renoncent à traduire l'image (« t'entraîner hors limites »; « drive you off the track »; « trascinan- 
doti fuori dalla pista »), tout en suggérant le contexte, pour DC et JH. PT, qui s'autorise un calque au v. 1466, avec « dicaste », peu clair pour le lecteur non helléniste, a aussi une belle trouvaille au v. 1084: « bureaucrates » pour hupogrammateôn. Autre anachronisme chez $\mathrm{JH}$, mais exceptionnel: les "green berets » pour les casques (pèlèkas, v. 1017).

Enfin, le domaine du corps (scatologie et obscénité comprises) est une composante essentielle de l'image comique du monde. Les divergences à ce propos révèlent clairement des orientations différentes chez les traducteurs. Nous essaierons d'avoir une vue d'ensemble en recensant les passages qui se réfèrent à un corps non héroïque, quotidien, voire trivial. ${ }^{6}$ Sur ces dix cas, nous laisserons de côté les v. 1070 et 1423 pour lesquels les solutions des uns et des autres convergent, et le v. 1280 dont le caractère éventuellement obscène ( « reins » pour « testicules ») est sujet à débat. DC fait bien apparaître la dimension corporelle, mais sans invention particulière dans les jeux verbaux. JH en fait autant avec en plus une trouvaille au v. 1308 à propos de la muse d'Euripide, où le elesbiazen condense une allusion à la haute poésie lyrique et une référence à la pratique de la fellation: "she never gave throat to a Lesbian tune ». PT, lui, reste vague sur ce point et, pour le reste, propose par deux fois des euphémismes (v. 1043, pornas = « catins », littéraire et vieilli là où les autres parlent de « puttane », « whores », ou de « bordel »; v. 1074, prospardein eis to stoma = péter en plein dans la figure »); enfin PJ combine trois traductions atténuant la présence du corps (v. 1075, « merder sur le camarade », resémantisation problématique d'un verbe à sens abstrait - rater son coup, mal se tirer d'affaire -; v. 1078, proagôgous = « des pourvoyeuses », vieilli et ambigu; v.1179, kataptuson = " crache dessus », où l'on entend que l'objet du crachat est le discours et non, comme souvent dans le dialogue comique et selon l'interprétation des autres traducteurs, l'interlocuteur lui-même). Il a deux trouvailles heureuses: v. 1308, « cette Muse-là [...] ne pratiquait pas les pompes de Lesbos » et v. 1327-8, " des chants dressés par la femme / Dodécatechnique de Cyrène », avec le jeu sur « dodécaphonique ». On a donc affaire à une opposition à trois termes: d'un côté, DC et JH, au franc-parler peu inventif (à une exception près); de l'autre, PT, plus timide; et PJ qui, paradoxal, combine les plus forts taux d'euphémisation et d'inventions grivoises.

$\mathrm{Au}$ bout de cette étape, deux grandes tendances se dégagent: DC et JH mettent bien en relief les traits comiques de la langue de l'original; PT et PJ creusent moins l'écart avec la langue tragique. Mais, il faut y revenir, ce qui est donné à entendre ici, ce n'est pas une mais bien deux langues tragiques.

Euripide (v. 1129-74) dénonce chez son rival la redondance, en débusquant dans les premiers vers de ses pièces quantité de pléonasmes ou de synonymes. De fait, c'est

\footnotetext{
6 V. 1043, 1070, 1074, 1075, 1078, 1179, 1280, 1308, 1327-8, 1423. Sur le jeu à propos du " pied » aux v. 1323 et suivants, voir ci-dessous, p. 8. Notre perspective, nécessairement synoptique, laisse de côté la question du moment où interviennent les propos obscènes dans la comédie. J. Robson en donne une analyse très fouillée dans ce volume, pp. 34-49.
} 
Euripide qui est ainsi, à son tour, défini comme esprit analytique dans la mesure où il ramène les mots à des concepts. Ce qui permet à Eschyle de le tourner en ridicule (v. 1198 s.): ou kat'epos ge sou knisô / to rhèm' hekaston, avec une image probablement empruntée à l'orfèvrerie: il s'agit de " gratter » les vers comme un métal avec une pierre de touche, pour les évaluer. ${ }^{7}$ Ici, JH et PT donnent bien l'impression de minutie quasi maniaque ( I certainly do not intend to pick away at your expressions word by word » et " Je ne vais pas dépiauter vers par vers / chacune de tes phrases »); il en va de même chez DC avec la tournure progressive (Non starò a grattarti (...) ogni parola), alors que la littéralité de PJ dessert plutôt l’idée ( « Je ne vais pas racler vers après vers / chacun de tes mots »).

Dans la «scène de la fiole » (v. 1197-1247), Eschyle se fait fort de "détruire » (diaphtheirein, v. 1200) les prologues de son rival à l'aide d'une petite fiole du type de celle où les athlètes, à la palestre, mettaient l'huile dont ils se frottaient. Il va donc, à chaque début de prologue de son rival, le couper et finir par la formule «lékuthion apôlesen » (« il a perdu sa fiole »), qui complète métriquement le vers interrompu. Quelle caractérisation d'Euripide est ainsi visée? Ce n'est pas une critique formelle, par exemple sur l'usage de la césure penthémimère ou du tribraque au quatrième pied dans les trimètres iambiques, puisque, comme on l'a souvent noté à la suite de Radermacher, ${ }^{8}$ ces phénomènes sont à peu près aussi fréquents, voire plus, chez Eschyle et Sophocle. ${ }^{9}$ Il s'agit plutôt de dénoncer la trivialité des objets traités par Euripide, lorsque l'insertion dans son discours d'une expression délibérément terre à terre, contrastant avec le style élevé du langage et la noblesse des héros évoqués, « casse » ses vers tout en s'y adaptant pour mieux les ridiculiser. ${ }^{10}$ Ici, toutes les traductions jouent évidemment de la répétition avec son effet de refrain et rendent l'accélération qui fait arriver de plus en plus tôt l'antilabè. PJ, en plus, utilise un hémistiche d'alexandrin: " égara sa gonflette », tout en cherchant à suggérer (de façon peut-être forcée, et peu claire) un sens obscène assez probable. ${ }^{11}$

Ainsi, la virtuosité d'Aristophane ne se limite pas à introduire la langue tragique dans l'univers de la comédie, ni même à exhiber les différences entre les styles

7 Cf. J. Taillardat, Les Images d'Aristophane. Etudes de langue et de style (Paris: Les Belles Lettres, 1965), p. 456 et note.

8 L. Radermacher (ed.), Aristophanes' "Frösche », Akad. der Wissenschaften in Wien, Phil.-histor. Klasse, 198. Band 4. Abhandlung, 1921, ad loc.

9 Pour l'analyse du vers appelé, justement d'après notre passage, « lécythion » ou « euripideion ", cf. Johannes M. Van Ophuijsen (trad. et comm.), Hephaestion on Metre (Leiden: Brill, 1987), p. 70 (H. VI.2). Sur les occurrences respectives chez les trois tragiques du tribraque au quatrième pied, cf. les statistiques in Dietmar Korzeniewski, Griechische Metrik (Darmstadt: Wissenschaftliche Buchgesellschaft, 1968), p. 55.

10 C'est l'effet repéré par Rau, Paratragodia (ci-dessus, note 1), p. 16, sous le nom de « Auflösung », ou « désintégration».

11 Sur ce point, voir avant tout l'article de J. Henderson et les autres sources citées par K. J. Dover dans son édition de la pièce, pp. 337-9. 
d'Euripide et d'Eschyle. Ces oppositions s'intègrent à un processus scénique plus général: l'agôn, ou joute, point culminant attendu de la comédie où s'exacerbe la tension entre deux personnages (qui ne sont pas ici, exceptionnellement, des protagonistes présents dès le début du drame).

\section{Poétique de l'agôn}

En effet, l'exposé sur les poétiques des deux auteurs et le débat critique sur leurs œuvres prennent la forme d'un affrontement entre deux personnages, caractérisables par un èthos, des stratégies de discours et une capacité à mener l'attaque ou à y riposter. ${ }^{12}$ Ces différents points sollicitent l'activité interprétative des traducteurs, sans parler des défis de traduction qui leur sont lancés.

Nombreuses sont les antithèses qui opposent Euripide et Eschyle: le poète des femmes (Phèdre, Sthénébée) et le chantre des hommes (Achille, Teucer); le quotidien contre la guerre; la langue de tous les jours ou un langage obscur; l'aulos ${ }^{13}$ (v. 1264) ou les ostraka (v. 1305). La traduction ne peut ici que reprendre ces oppositions, le seul choix étant d'attirer ou non l'attention sur la dernière par des didascalies, ${ }^{14}$ comme le font DC et PT, à l'inverse de JH et de PJ (qui conserve toutefois la seconde indication). De même, certains préfèrent désigner les instruments par leur nom grec (PT: aulos), des gloses (DC: «flauto doppio ») ou des équivalents modernes (« nacchere » pour ostraka, que Judet et PT rendent par « castagnettes »). Par ailleurs, le portrait d'Eschyle insiste volontiers sur sa propension à s'emporter, un peu comme si l'auteur devait ressembler à son œuvre, comme le suggèrent les v. 900-4. Il arrive aux traducteurs d'y insister: ainsi, PT et son « mille tonnerres » au v. 926, qui peut aussi bien sembler incongru, par emprunt au monde d'Hergé, que pertinent, puisqu'il entre dans un réseau d'images. Eschyle est aussi massif, entier: en témoigne l'effet-bélier de ses débuts de réplique repris aux v. 1183 et 1188, Ma ton Di’ou dèt' [...], rendu efficacement par JH (« Certainly [...] not », repris), par DC (« No davvero, per Zeus! », repris) et par PJ (« Mais pas du tout, nom de Zeus », deux fois), un peu perdu chez PT qui ne répète pas textuellement («Oh que non, grand dieu! »; « Penses-tu! Mais non, grand

12 On verra que l'èthos des deux poètes est construit d'après leurs œuvres, selon un procédé systématique chez Aristophane, que l'on retrouve par exemple dans les Acharniens et les Thesmophories.

13 L'aulos est un instrument à anche double, proche du hautbois. Voir André Schaeffner, Origine des instruments de musique. Introduction ethnologique à l'histoire de la musique instrumentale (Paris: Payot, 1936; rééd. EHESS, 1968, 1980, 1990), p. 270, et surtout M.L. West, Ancient Greek Music (Oxford University Press 1992), pp. 81-107. Voir aussi Annie Bélis, Les Musiciens dans l'Antiquité (Paris: Hachette, 1999).

14 Sur les questions que pose l'usage plus ou moins étendu des didascalies chez les traducteurs, voir les précieuses observations de J.M. Walton dans sa contribution à ce volume (pp. 1052-3). 
dieu »). Jusqu'où les traducteurs sont-ils prêts à aller dans cette représentation d'un Eschyle colérique? C'est là une des questions que pose l'attribution du v. 947: Eschyle, dans son emportement, peut-il attaquer Euripide sur son origine? C'est ce que pensent JH, DC et PJ. PT est d'un avis contraire et met la réplique dans la bouche de Dionysos. Ce faisant, les traducteurs s'autorisent évidemment de traditions manuscrites divergentes. Mais cela prouve une activité interprétative dès l'étape des copistes, qui, eux aussi, se font une certaine idée du caractère de chaque personnage, ainsi que le montre DC dans ses notes aux v. 942, 1130 et 1320-2, notamment.

On vient de le voir sur un détail, l'animation de la dispute entre les deux auteurs tragiques est inégalement rendue. L’image de la morsure au v. 861 (daknein, daknesthai) doit pourtant être prise au sérieux: elle ramène le duel des poètes à un affrontement entre animaux, entre chiens peut-être; elle se prolonge à plusieurs reprises (avec l'idée sous-jacente du flair d'un limier v. 893, ou la mention des dents v. 927); elle resurgit à la faveur d'un possible jeu de mots sur melè au v. 1262 (les « chants » mais aussi les « membres ») et d'une rémanence du sens premier de temnô ( " couper », " trancher ») dans le suntemnô (" résumer »), au même vers, à en croire les analyses de DC. Ainsi l'échange est vif, agressif, et il faut se garder de l'affadir. Les deux adversaires se coupent la parole, disloquant l'unité du vers: le phénomène n'est pas dur à restituer. Il leur arrive également de retourner à l'adversaire un de ses termes, avec un raffinement efficace dans la violence. Tel est l'échange aux v. 10446: Eschyle commence par reprendre le verbe epeimi, employé par Euripide dans le sens de « être associé à » (pour dire, en substance, " Aphrodite n'a rien à voir avec toi »); puis il réutilise le préfixe verbal epi comme préposition avec une nette idée d'hostilité («Aphrodite te poursuit »). A défaut de pouvoir user des mêmes moyens lexicaux et verbaux, certains traducteurs (JH, DC) arrivent à rendre la vivacité de la riposte, là où les autres sont à la peine. Mettre le discours de l'autre en pièces: tel est peut-être le but de chacun des deux auteurs tragiques lorsqu'ils en arrivent à proposer des pastiches de leur adversaire. Au plan herméneutique, on peut discuter de la question de savoir si le collage de vers « à la manière de » vise à souligner le manque d'originalité de procédés répétitifs ou à mettre en avant ce qui apparaît à chaque auteur comme une incohérence radicale dans l'univers esthétique de son rival; on peut lire l'affirmation de la banalité d'un style homogène, ou, au contraire, l'incongruité d'éléments hétérogènes. Au plan pratique, pour les traducteurs, il faut choisir entre une syntaxe qui garde un minimum de cohérence, ou un texte qui assume la fragmentation, jusqu'à l'absurde. Lorsque Euripide imite le style d'Eschyle (v. 128595), PT et PJ conservent une certaine continuité syntaxique, contrairement à DC et $\mathrm{JH}$. Quant à la restitution du morceau de bravoure d'Eschyle inventant une monodie sur le modèle euripidéen, les attitudes des traducteurs sont, là encore, variées. PT et DC découpent le passage (v. 1331-63) en strophes qui, paradoxalement, introduisent un principe d'intelligibilité, donc de continuité. Les mots de liaison, en grec, signalent les différents moments du chant et l'on peut, par exemple, préférer « now » (au v. 1356a) pour rendre all', à un possible « but », ou au «mais » adopté par PJ, car la 
succession des pensées n'obéit pas à la pure logique, mais plutôt à un principe de fluctuation émotionnelle, ce que DC rend par l'adverbe « orsù », invitation soustendant les vocatifs, en même temps qu'elle amorce un mouvement conclusif.

Nous venons de parler d'un morceau de bravoure d'Eschyle. Il est, en effet, permis de penser que sa performance (argumentative, imitative ...) est supérieure à celle d'Euripide. A tel point qu'on peut lire l'ensemble de la scène sur le modèle du renversement du rapport de force. On aurait au début un Euripide qui mène l'attaque, ce qui est d'ailleurs explicable par le fait qu'il est challenger. Puis un Eschyle qui reprend la main et se révèle plus astucieux: n'est-ce pas lui qui suggère de passer à la pesée (déjà évoquée en 797) et, surtout, qui invente l'épisode du lèkuthion? Ou qui, au v. 1343, introduit un coq dans son pastiche d'Euripide lequel, au v. 935, ironisait sur la présence du volatile dans une tragédie (d'Eschyle, bien sûr)? Schéma du retour à l'envoyeur... Eschyle personnage d'Aristophane est drôle, vif; il a le sens de la répartie et du second degré. Par exemple, au v. 1323, il joue sur le double sens (physique et métrique) du mot podon. ${ }^{15}$ Il n'est pas sûr qu'aucun de nos traducteurs réussisse à rendre l'effet. Certains, comme PT, recourent à une note. Et l'on en vient à regretter le spirituel « Tu vois ça, ces pieds en l'air? » de Victor Debidour ${ }^{16}$ !

En effet, il ne faudrait pas oublier que l'affrontement entre Eschyle et Euripide ne se limite pas à un aspect verbal. Il est mis en espace, en musique et dans une moindre mesure chorégraphié, conformément à la curieuse alliance de termes du v. 896 logôn emmeleia. Les traductions rappellent très inégalement à leurs lecteurs que le texte qu'ils ont sous les yeux était destiné à la scène. Certes les manuscrits comportaient quelques didascalies (ou parepigraphai), le plus souvent reprises dans les versions modernes, au v. 1263, par exemple. Mais plusieurs passages demeurent obscurs si l'on n'imagine pas une gestuelle, un a parte, ou des accessoires. Par exemple, aux v. 1323-4, la question répétée d'Eschyle signifie-t-elle une pure insistance, ou que le poète tragique la pose successivement à Dionysos et à Euripide (ou l'inverse)? Pour PT, Eschyle s'adresse par deux fois au dieu du théâtre; pour JH et DC, il se tourne vers Euripide, tandis que PJ suppose qu'il prend Dionysos à témoin de ce que vient de reconnaître Euripide. De plus, comment intervenir pour éclairer l'aspect scénique du texte? Par des notes, qui ne seront pas toujours lues, et peuvent être formulées sur le mode hypothétique? Ou par des didascalies, qui n'échapperont pas au lecteur et lui paraîtront plus assurées? JH multiplie les indications: il mentionne la présence de trois sièges au moment de l'entrée de Pluton, Eschyle et Dionysos sur le plateau (p. 139), ou

15 Pour une interprétation qui corrobore le sens physique tout en discutant la nature du sens métrique (kôlon plutôt que "pied” dans l'acception moderne), voir Alessandra Lukinovitch, Mélodie, mètre et rythme dans les vers d'Alexis. Le savoir-faire d'un poète comique (Grenoble: Jérôme Millon, 2009), p. 29 et note 27. Le même double sens se trouve déjà dans les Thesmophories, vers la fin du chant d'Agathon (v. 120-1).

16 Aristophane, Théâtre complet, tome 2, texte traduit, présenté et annoté par V.-H. Debidour (Paris: Le Livre de Poche, 1966), p. 353. 
celle d'outils de mesure (dont une balance) qui serviront plus loin dans la pièce, page 137; il attire l'attention sur l'encens dont il sera question au v. 888, en décrivant le rituel juste avant le chant du chœur (p. 145). Les deux premières didascalies sont moins assurées que la troisième et pourtant elles ont toutes le même statut aux yeux du lecteur. PT, lui, répartit les informations entre la traduction elle-même et les notes. Par exemple, dans le corps du texte, juste avant le v. 830, «Dionysos, Eschyle et Euripide entrent en scène ", et une hypothèse, en note ("Ils sont peut-être accompagnés de figurants muets, parmi lesquels Pluton et Perséphone », p. 1279). A quoi s'ajoutent les précisions de la " Note sur la mise en scène » (p. 1266-9), notamment sur la distribution des rôles entre les quatre comédiens, le deutéragoniste et le quatrième acteur se partageant éventuellement le rôle de Pluton. Dans sa préface, DC dit à l'inverse se méfier du petit jeu des reconstitutions scéniques (p. XXI). Pour lui, il convient de ne se concentrer que sur " alcuni tra i più significativi di questi fatti spettacolari », sous peine de se perdre dans un labyrinthe. Pluton sort-il de son palais au v. 1414, ou a-t-il assisté à l'ensemble de la querelle entre Euripide et Eschyle? La question lui semble douteuse autant que peu pertinente, d'après la note 1 p. XXV. Reste que la dispute entre Eschyle et Euripide se déroule sous le regard de plusieurs publics: les spectateurs, silencieux, assis dans les gradins; peut-être un Pluton sur scène et silencieux; et, sur le plateau, différents témoins qui s'expriment (le chœur, Dionysos et l'adversaire).

Il s'agit en effet d'un combat arbitré, qui appelle le jugement. On peut, à ce sujet, s'interroger sur les éléments de formalisation du discours, sur les réactions du chœur et de Dionysos et enfin sur ce qui prépare et éclaire le jugement final.

La querelle entre Euripide et Eschyle obéit à plusieurs modèles formels qui se superposent, au premier rang desquels le procès et la joute poétique: les traductions nous alertent très inégalement sur le phénomène. Le vocabulaire juridique, tout d'abord, affleure aux v. 908, 922 et 1366, avec le verbe elenkhô (et ses composés), qui peut signifier « convaincre d'une faute, d'un tort » ou, dans un emploi moins spécialisé, « blâmer, accuser ». Même remarque pour le verbe krinein (aux v. 1416 et 1466) que l'on peut comprendre au sens fort comme " rendre un verdict » ou dans l'acception plus lâche de " décider ». Quant au prosopheilôn du v. 1133, faut-il y lire l'idée d'une mise à l'amende comme JH, qui propose la traduction « liable for »? De fait, ce traducteur recourt volontiers à des expressions juridiques ${ }^{17}$ : outre l'exemple cité, relevons « please render your verdict » au v. 1466, « death penalty » au v. 951 et « the only real test » (avec le sens probable de « critère ») au v. 1366. PJ choisit le plus souvent des termes à la frontière entre un sens intellectuel et un sens juridique: ainsi de " réfuter » au v. 922, ou d' " établir » au v. 908, qui devient " établir le vrai » au v. 1366. Le « tu n'es pas quitte » au v. 1133 évoque toutefois l'idée judiciaire d'acquit-

17 Ce qui n'étonnera pas, venant d'un chercheur s'intéressant tout particulièrement au rapport entre langue comique et langue rhétorique, ou juridique. 
tement et ne se situe plus strictement au plan intellectuel. Les champs lexicaux chez PT et DC sont moins spécialisés. On trouve chez eux aussi bien des termes à valeur intellectuelle ( « je veux faire la preuve » et « dimostrando » au v. 908, ou « évaluer » et «fare la critica » au v. 1366) que des mots renvoyant à l'univers théâtral ( " smascherò » et, moins nettement, " confondre », au v. 922), ou encore un vocabulaire neutre (« dovresti decidere » et « veux-tu indiquer ta décision », au v. 1466), DC étant le seul à faire passer krinein en 1416 au plan affectif (« ti va bene »). Il est également le seul à faire apparaître la codification de la joute. ${ }^{18}$ Il est éclairant de comparer la traduction de mousikôtata au v. 873 par JH (« with integrity ») et par DC (« a regola d'arte »). Emprunt donc au vocabulaire de l'art et/ou de l'artisanat chez le traducteur italien, qui utilise aussi beaucoup de métaphores sportives (« siamo pronti » au v. 1381 et surtout « rivincita » en 1389, « ripresa » en 1401 et « gara » en 1407). Il y a là, clairement, l'idée d'une compétition réglée.

Celle-ci est soumise à l'évaluation du public. On sait qu'Eschyle l'emportera. Le chœur, d'abord neutre, marque sa préférence pour lui aux v. 1255 et suivants. Et le verdict de Dionysos permet à Eschyle de revenir sur terre, alors que le dieu du théâtre était initialement venu chercher Euripide. Quelles sont les étapes du renversement de situation? Comment les traducteurs (se) représentent-ils les alliances entre les personnages et leur modification? L'étude de la traduction des vocatifs, qui laissent une relative liberté, se révèle instructive. JH adopte volontiers un ton de sympathie décontractée lorsque Dionysos s'adresse à Euripide (« my man » en 1235, « my friend », en 952 et en 1227), ou en sens inverse ( « my man », en 1243). PJ risque un calque littéral en 1227 (« ô cher homme divin »), recourt à un « cher ami » (en 952 et 1243) qui sonne désormais comme guindé en français et à un « bon ami » en 1235, entre condescendance et enjouement. Les choix de PT ne sont pas très éloignés: un "mon tout excellent » en 1227, et un "mon cher » en 1235 bien cérémonieux, sauf si on le comprend au second degré, ce que la traduction par « l'ami», plus relâché, en 952 et 1243, peut inciter à faire. L'hétérogénéité des choix brouille la perception du lecteur: on verra plus loin que l'interprétation de " pauvre » en 853 est incertaine chez PT (sollicitude? supériorité?). DC, lui, maintient constamment une proximité affective entre le dieu du théâtre et Euripide (« caro mio » en 952 et 1227; « amico mio» en 1235, « mio caro » en 1243; à quoi répond un « mio caro » au v. 1243). Non seulement, en 1214, comme ses confrères (sauf PT), il conserve la première personne du pluriel par laquelle Dionysos s'associe à Euripide, mais il invente la réciproque en 1223 où son Euripide dit « manderemo » en parlant de Dionysos et de lui-même (le texte grec, lui, présente une troisième personne du singulier à la voix passive). Autre passage qui permet de voir comment les traducteurs pensent la relation entre les personnages, le

18 Sur les rapports entre cette scène des Grenouilles et le Certamen d'Homère et d'Hésiode, voir Marina Cavalli, « Le Rane di Aristofane: modelli tradizionali dell'agone fra Eschilo ed Euripide », in: Fabrizio Conca (éd.), Ricordando Raffaele Cantarella. Miscella di studi, «Quaderni di Acme » 36 (Milan: Università degli studi, 1999), pp. 83-105. 
v. 1209: Dionysos est alors excédé par le lèkuthion. JH et DC insistent sur la communauté d'intérêts entre Dionysos et Euripide, respectivement par « to hell with it » et « alla malora! ». Les traductions de PJ (« qu'il en gémisse! ») et de PT (« il va s'en mordre les doigts ») sont moins expressives et mettent en avant la défiance de Dionysos à l'égard d'Eschyle plutôt que l'identité des vues entre le dieu et Euripide.

Pourtant, Dionysos tranche en faveur d'Eschyle au v. 1471. On verra plus loin que la formulation utilisée fait question. Intéressons-nous pour l'instant aux temps forts sur le chemin de la décision. Au v. 1413, Dionysos exprime son hésitation dans une antithèse dont le sens exact est discutable (sophos s'applique-t-il à Eschyle, comme le pensent la plupart des commentateurs modernes? ou à Euripide, ce que comprenait Aristarque?). Il en va de même avec l'antithèse du v. 1434: sophôs/ saphôs. Avant ces deux mouvements d'hésitation, le v. 1415 n'est pas compris de la même manière par les traducteurs, à la faveur d'une ambiguïté du grec: ton heteron peut signifier aussi bien "l'un des deux » que "l'autre des deux ». JH et PJ préfèrent le premier sens (« and what if I do reach a verdict? »; "l'un des deux »), en supposant donc que Dionysos s'informe simplement auprès de Pluton des conséquences de son choix, quel qu'il soit. PT suggère déjà que Dionysos penche pour Eschyle: « et au cas où je choisirais l'autre? ». En effet, il paraît difficile de ne pas entendre « l'autre que celui que j'étais venu rechercher », c'est-à-dire Eschyle, et seul le conditionnel maintient un certain suspens. Suspens précisément refusé par DC qui glose son « quell’altro? » par une didascalie («accennando a Eschilo»). Par conséquent, le traducteur italien anticipe très (trop?) tôt le moment du choix. Il ne l'explique d'ailleurs pas dans les mêmes termes que ses confrères. En 1468, Dionysos dit suivre la volonté de sa psuchè: les deux traducteurs français traduisent par «âme »; JH par « soul », tandis que DC préfère « animo » à " anima », que l'on attendrait plutôt. Si « soul » et « âme » prennent une couleur paratragique et font entendre un Dionysos qui cesse d'être, à cet endroit, un personnage comique, s’il y a également peut-être un jeu avec l'orphisme, dans le contexte de la catabase, on peut estimer que DC, sans doute parce qu'il accentue volontiers la dimension comique (familière, impulsive, etc.) de Dionysos, échoue ponctuellement à faire entendre le phénomène. ${ }^{19}$

Car tous les personnages ont plusieurs langages dans Les Grenouilles : les auteurs tragiques ont un style identifiable, tout en pastichant celui de l'adversaire, à l'occasion, et en entretenant un dialogue comique; Dionysos a une dimension bouffonne qui sous-tend « le caractère comique » des scènes, mais c'est aussi un amateur de poésie, qui s'essaie par instants à imiter le ton tragique. Cette polyphonie à l'intérieur même des propos de chaque personnage est particulièrement dure à saisir et à restituer. Elle exprime aussi la manière dont chacun a reçu la voix de l'autre. Elle pose, si l'on préfère, la question de l'écoute comique de la tragédie.

19 A moins que l'on ne fasse entrer en ligne de compte l'acception, assez rare et littéraire, de animo comme « âme » au sens spirituel, voire eschatologique. 


\section{L'écoute comique}

La voix de chaque personnage est à la fois susceptible d'être comique et tragique; chaque discours est traversé par celui des autres: cela met l'herméneute qu'est d'abord le traducteur face à un certain nombre de problèmes. Pour aller vite, ramenons ceux-ci à trois: l'orchestration des voix, le repérage de l'ironie, la visée critique du rire.

Dans une comédie où le langage est pris pour objet et où deux auteurs tragiques tantôt parlent conformément à leurs écrits, tantôt imitent par raillerie la parlure de l'adversaire, le tout en devenant personnages comiques, et en interférence avec le dieu du théâtre, indissociablement caricature bouffonne et critique avisé, la simple question de savoir qui parle devient complexe. Le fait est que, dès la tradition manuscrite, la répartition des répliques témoigne d'une relative indistinction des voix, du moins dans la perception du copiste, puis du philologue. Afin de donner une idée du processus, limitons-nous ici aux cas où Dionysos dit quelque chose que l'on peut également attribuer à l'un des deux poètes tragiques. Examinons les choix de PT, souvent singuliers, et, pour la clarté de l'analyse, distinguons les passages où (par rapport aux autres traducteurs) il substitue un locuteur à un autre, et ceux dans lesquels il scinde une même réplique entre deux personnages.

Au v. 947, nous l'avons vu, PT est le seul à penser que c'est Dionysos qui parle (et non Eschyle); en 1162, il « rend ce vers à Euripide comme le font tous les manuscrits ", que ne suivent pourtant aucun des trois autres traducteurs (lesquels estiment que c'est Dionysos qui s'exprime); aux v. 1323-4, PT attribue la réplique à Dionysos et non à Euripide, contrairement à DC et à JH (PJ choisissant une solution médiane avec une première réplique pour Euripide et une deuxième pour Dionysos); au v. 1399, Dionysos joue un coup à la place d'Euripide dans toutes les traductions, sauf chez PT, pour qui c'est Eschyle qui intervient. (Par conséquent, ici, la proximité entre Dionysos et Euripide est moindre, puisque le dieu ne protège pas le poète, ce qui permet l'indécision sur l'interprétation de " pauvre », dont nous parlions plus haut.); chez PT (et DC, qui s'en explique), le v. 1130 est prononcé par Eschyle, qui proteste, alors que, chez JH et chez PJ, Dionysos se fait le porte-parole de ce dernier.

En 1023-4, PT scinde une réplique en imaginant que Dionysos prend la suite d'Euripide. Le cas du v. 1418 est proche du précédent, sauf qu'il implique Eschyle: allant contre la majorité des manuscrits, PT distribue une réplique entre Eschyle et Dionysos.

Qu'en conclure? Dans cinq cas sur sept (v. 947, 1023, 1130, 1162, 1399), les modifications proposées par PT accroissent la triangulation avec un Dionysos qui parle globalement moins, parce que les échanges deviennent plus vifs avec un Eschyle et un Euripide qui s'emparent de la parole au beau milieu d'une réplique. A en juger par la note page 1287, relative aux v. 1436-66, on peut penser que PT poursuit précisément cet effet. Par ailleurs, dans quatre cas sur sept, les changements dans l'attribution ou le découpage du texte montrent que, pour PT, la voix de 
Dionysos peut plus facilement se confondre avec celle d'Euripide qu'avec celle d'Eschyle, à tel point, du reste, que le traducteur n'imagine pas, au v. 1130, que Dionysos puisse faire une objection à Euripide. Toutefois, au v. 947, il suppose que Dionysos attaque Euripide (ce qui n'arrive jamais chez DC) et qu'en 1399 il ne lui souffle pas une solution. Ce qui peut donner l'impression d'une proximité de fond entre Dionysos et Euripide, laissant contradictoirement place à des mouvements agressifs. Il est possible, toutefois, qu'en mettant le v. 947 dans la bouche de Dionysos, PT ait simplement voulu éviter de faire dire une mesquinerie à Eschyle, bien qu'il attribue une platitude à ce dernier en 1130. (Nous retrouvons ici l'idée d'un èthos prêté aux personnages.)

Mais suffit-il que Dionysos prenne fait et cause pour Euripide pour que la discussion tourne à l'avantage du poète? L'issue de la scène nous prouve que non. Ce qui pose la question de l'ironie au premier niveau d'énonciation (celle des personnages) ainsi qu'au second (celle de l'auteur en direction du lecteur/spectateur). ${ }^{20}$

Dans les v. 980 et suivants, il est presque certain qu'Aristophane souhaite que nous ayons une perception amusée de l'enthousiasme de Dionysos pour les innovations poétiques d'Euripide. De même, en 1181, l'admiration du dieu (toujours pour Euripide) a quelque chose de maladroit et de ridicule. Et que faire de la mise en garde adressée à Eschyle par le chœur v. 992 et suivants? Maintient-elle, ou non, une distance ironique? Si oui, comment les traducteurs peuvent-ils nous y rendre sensibles? Par l'emphase? Par de subtiles dissonances?

Même l'ironie au premier plan énonciatif n'a rien de l'évidence. Suivons, cette fois, la traduction de DC, qui souligne le soutien de Dionysos à Euripide mais aussi la supériorité d'Eschyle, malgré sa mise en minorité. Ainsi, au v. 1170, Dionysos abonde dans le sens d'Euripide, comme l'atteste la répétition de « avanti » (répétition sans équivalent en grec), mais on peut également estimer qu'il est du même coup à la traîne, ce que préparerait son aveu au v. 1169. DC, plus que tout autre, fait le pari d'une ironie entre les personnages. En l'occurrence, il restitue la moquerie d'un Eschyle à l'égard d'Euripide. Il suppose qu'Eschyle coupe la parole à son rival, pour feindre d'aller dans son sens, mais en réalité pour lui retourner une interprétation ironique de ses propos (sur le modèle familier du « je ne te le fais pas dire! »). Ainsi les deux phémi kagô, des v. 954 et 958, sont compris comme des interruptions ménageant une perception au second degré des affirmations d'Euripide. Le phénomène est encore plus visible dans la tirade où Euripide fait son propre éloge (v. 937 et suivants), tirade dont DC répartit le contenu entre Euripide et Eschyle: en quelque sorte, l'autopromotion d'Euripide alimente la critique d'Eschyle. Ce principe généralisé d'ironie indique que DC adopte globalement le point de vue d'Eschyle, et d'un Eschyle particulièrement facétieux. Cette posture interprétative, sensible dans la traduction, est confirmée dans les notes.

20 Selon l'analyse d'Anne Ubersfeld, Lire le théâtre (Paris: Editions sociales, 1978), pp. 250-4. 
Pour DC, donc, le rire d'Eschyle est aussi celui d'Aristophane. Il est même ici le moyen de l'analyse du style d'Euripide. Mais est-ce toujours le cas? Y a-t-il constamment un sens critique du rire? Ou bien le jeu comique peut-il être gratuit? Et quand il se concilie avec le sérieux des considérations poétiques, est-il univoque, vise-t-il toujours le même poète? Ou atteint-il chacun tour à tour? Serait-il, même, le moyen de l'ambiguïté, et d'un jugement indissociablement laudateur et critique sur chacun des deux auteurs tragiques?

Prenons d'abord la mesure des effets de la double énonciation et de la présence cachée d'Aristophane derrière ses personnages. Si, nous l'avons vu, Eschyle et Euripide sont caractérisés, aussi bien comme personnes que comme auteurs, avec une indéniable visée référentielle de ces portraits, il ne faut pas oublier qu'ils sont tout autant devenus personnages comiques, à travers lesquels s'exprime Aristophane. En clair, Eschyle a un style eschyléen (par exemple dans son invocation à Déméter aux v. 885 et 886), mais il parle à l'occasion comme Aristophane. Ainsi son résumé de la tragédie d'CEdipe, aux v. 1188 à 1195, en propose une vision comique: le fils de Laïos est décidément trop nigaud (épouser une vieille qui de plus est sa mère!). Il importe que les traducteurs rendent la faconde du personnage et sa drôlerie. On peut à cet égard saluer la trouvaille de PT, en 1015, et son « agorateurs ». Autrement dit, une constante instabilité affecte le langage des personnages: il appartient à la traduction de rendre cette hétérogénéité, ces ruptures qui sonnent comme des incongruités. Dans sa monodie à la manière d'Euripide, en 1360, Eschyle emploie un diminutif pour parler des kuniskas d'Artémis: le « cagnette » et les « chiots » (PJ) donnent une idée du phénomène; les «toutous » aussi, mais au prix d'une exagération familière chez $\mathrm{PT}$, tandis que la surprise lexicale disparaît chez $\mathrm{JH}$, sauf à entendre « bitch » dans son acception injurieuse, avec un déplacement sémantique, du même coup. Encore faut-il noter qu'on peut mener la même analyse en ce qui concerne la langue d'Euripide. Celui-ci devient pur personnage aristophanesque dans le combat naval imaginé aux v. 1437 et suivants. Il est aussi capable d'une belle imitation moqueuse d'Eschyle, au v. 963, et raille le goût de celui-ci pour les mots composés (kôdônophalaropôlous). Quant au chœur, qui adopte souvent une langue paratragique, il glisse à l'occasion des éléments comiques dans ses pastiches (par exemple le stômullete du v. 1311). L'hétérogénéité du langage est de mise partout.

Mais alors, si tout le monde se moque de tout le monde, comment déterminer le sens du texte? Les amateurs de certitude peuvent faire valoir qu'un jugement est rendu en faveur d'Eschyle, ce qui lèverait tout doute. Ceux qui supposent qu'un auteur ne varie jamais et qui déduisent le sens d'un texte en le confrontant avec d'autres textes du même auteur peuvent remarquer les points de rencontre entre les répliques d'Eschyle et les attaques menées ailleurs par Aristophane contre Euripide, dans Les Thesmophories, par exemple. A cela (ou ceux-là), on peut faire quelques objections. Il ne suffit pas de gagner pour avoir raison. La preuve en est, à nos yeux, aux v. 1163-5, où Eschyle trouve à répondre à Euripide, où il justifie ce qui pourrait apparaître comme un pléonasme et s'attire alors un compliment de Dionysos. N'a-t-on 
pourtant pas vu, en 980 et suivants, que l'admiration du dieu était parfois à double tranchant et que son enthousiasme naïf équivalait, en réalité, à un désaveu, quand on change de plan énonciatif? Dans le cas d'espèce, faut-il exclure que, au-delà même du différend entre les deux auteurs tragiques, Aristophane prenne pour cible les distinctions (d'Eschyle) à la Prodicos? On aurait ici un clin d'œil de l'auteur à son public, par-dessus la tête des personnages.

Autrement dit, il convient plutôt d'assumer la relation du rire à la polysémie. Dionysos, nous n'avons cessé de le voir, est divisé; il hésite. En 1471, il choisit Eschyle mais il continue de parler comme Euripide, plus que jamais même (1471 et 1477-8). On peut décider de réduire la contradiction en voyant une raillerie dans l'imitation: Dionysos passerait alors une alliance totale et entière avec Eschyle. Mais on peut souhaiter prolonger l'indécision de Dionysos et ses contradictions internes, qui l'amènent à manier l'antithèse (v. 1471) et le questionnement (v. 1477-8). Dans cette perspective, il faut veiller à ménager la possible ambiguïté de ces formules d'adieu et de désaveu: s'agit-il d'un raffinement dans la cruauté (on retourne contre l'autre ses propres formules)? ou d'un dernier hommage (décidément, Dionysos a plaisir à parler la même langue qu'Euripide)? Tout dépend de la part que l'on accorde à la situation d'énonciation (verdict, adieu) dans l'interprétation de l'énoncé. Si l'on est prêt à admettre que l'énoncé et l'énonciation peuvent être en tension, alors Dionysos peut préférer la langue d'Euripide à celle d'Eschyle (jeu de mots sur glôtta, en 1471), tout en revenant sur terre avec l'auteur des Perses. Autre argument en faveur d'une interprétation polysémique du rire: le goût de la comédie pour les métaphores concrètes, qui suggèrent autant qu'elles disent. Elles désignent, elles montrent une direction interprétative mais ne verrouillent pas le sens. D’après Les Grenouilles, Eschyle a un style lourd, pesant, qu'Euripide a jugé bon de mettre à la diète; mais c'est pour cela qu'Eschyle remporte l'épreuve de la balance! Comment comprendre cette métaphore de la lourdeur: emphase insupportable ou poids des propos? Peut-être convient-il que les traducteurs ne tranchent pas quand ils ont affaire à des métaphores et qu'ils conservent la polysémie de ces trouvailles verbales. En 1154 et 1178, PT a probablement tort d'utiliser un vocabulaire trop spécialisé (" pléonasme »). ${ }^{21}$ De même, en 1178 toujours (mais dans la deuxième moitié du vers: è stoibèn idèis), le « zeppa » de DC est sans doute trop précis, même si l'acception technique du terme en italien peut se comprendre dans un contexte littéraire. JH semble davantage dans le ton, avec un terme au champ sémantique large: « padding ».

L'écoute comique de la tragédie ne peut être réductrice. La comédie ne peut adopter sans réserve un auteur tragique, sous peine de se renier. La moquerie du v. 1005 sur le tragikon lèron vise nécessairement les deux auteurs. A l'inverse, au

21 Ce registre pose la question, soulevée par J. Taillardat, de l'existence à l'époque en grec d'un vocabulaire spécialisé de la critique littéraire et de sa relation aux métaphores d'Aristophane. Voir son analyse de la pesée, Les Images d'Aristophane (ci-dessus, note 7), pp. 467-70. 
v. 1370, pourquoi deviennent-ils conjointement objets d'admiration (epiponoi g' hoi dexioi)? Sans doute grâce à leur dimension comique: à cet endroit de la scène, devenus personnages comiques à part entière, ils mettent en place la pesée des vers. Et même si Eschyle gagne la mise parce qu'il fait le plus rire, le texte des Grenouilles œuvre pour lui et en même temps contre lui, tout comme il va contre Euripide et pour lui. 22

Après une analyse de différentes expériences de traduction, Umberto Eco concluait récemment: «Interpréter signifie faire un pari sur le sens d'un texte. Ce sensqu'un traducteur décide d'identifier-n'est recélé dans aucun monde des idées [...]. C'est le résultat d'une série d'inférences que partagent ou non d'autres lecteurs. ${ }^{23}$ "

$\mathrm{Au}$ terme de notre propre parcours, nous faisons le même constat, qui, de fait, a aussi déterminé notre démarche, prises que nous étions dans la circularité herméneutique. En effet, notre méthode a consisté pour l'essentiel à croiser les approches: lire les traductions comme des textes autonomes afin de repérer leurs partis pris, leur esthétique, leurs points aveugles éventuels, leur lisibilité aussi; les comparer entre elles afin de mieux dégager leur spécificité et, ce faisant, revenir périodiquement au texte grec, pour y cerner aussi bien les difficultés philologiques de tout ordre (sémantique, grammatical...) que des questions de poétique (interprétation de la double énonciation, mise en espace, etc.).

Il nous est apparu que la lecture des traductions s'apprend. On y découvre en acte les exigences d'un exercice périlleux, dont le résultat est toujours un artéfact induisant une interprétation. On y mesure l'écart entre un horizon d'attente (un Aristophane comique, pittoresque, philosophique, etc.) et les choix opérés par le traducteur. Ce qui permet d'identifier certains des critères qui font juger une traduction bonne ou mauvaise.

Et surtout, ces examens croisés ramènent au texte, dont ils déploient les strates, les tensions sémantiques, les virtualités. Invitation du lecteur du texte original à différer l'arrêt du sens et à laisser se démultiplier les pistes interprétatives, puisqu'il n'est pas tenu de fixer mentalement les choses. Au bout du compte, ces jeux comparatifs renforcent une perception polyphonique de la pièce; impossible désormais de voir en Aristophane un donneur de leçons, politiques ou esthétiques. Mais nos quatre traducteurs donnent très inégalement l'image d'un Aristophane capable de (faire) penser par le rire...

22 Pour une interprétation un peu différente du jugement de Dionysos et de ses implications quant aux choix poétiques et politiques d'Aristophane, voir dans ce volume la contribution de M. Alonge, p. 82-93.

23 Umberto Eco, Dire presque la même chose. Expériences de traduction (2003), traduit de l'italien par Myriem Bouzaher (Paris: Grasset, 2006), p. 180-1. 